\title{
Ocular manifestations in a patient with de novo Fabry disease
}

\author{
You Hyun Lee ${ }^{1}$, Kyu Young Shim', Sung Bae Park ${ }^{2}$, Yu Cheol Kim ${ }^{1}$ \\ Departments of ${ }^{1}$ Ophthalmology and ${ }^{2}$ Internal Medicine, Keimyung University School of Medicine, Daegu, Korea
}

Fabry disease (FD) is an X-linked, recessively inherited, rare, progressive, disorder of glycosphingolipid metabolism affecting multiple organs resulting in organ dysfunction. It is rare to find only one FD affected subject with a de novo mutation. Here we report a case of a 41-year-old Asian male diagnosed with de novo FD. Comprehensive ophthalmological evaluation was performed using slit lamp, color fundus photography, optical coherence tomography, fluorescein angiography, and indocyanine green angiography. On slit lamp examination, cornea verticillata and slightly tortuous, and aneurysmal dilatation of inferior bulbar conjunctival vessels were observed. Other imaging modalities showed unremarkable findings. Cornea verticillata and inferior bulbar conjunctival vascular abnormalities may be detected earlier than other ocular abnormalities in de novo FDs like hereditary FDs.

Keywords: Cornea verticillata; De novo mutation; Fabry disease

\section{INTRODUCTION}

Fabry disease (FD) is an X-linked, recessively inherited, rare, progressive, disorder of glycosphingolipid metabolism affecting multiple organs and resulting in organ dysfunction [1]. The incidence of FD is 1:117,000 live births and among them, very few "de novo" mutations have been detected [2,3]. The most frequently reported clinical manifestations of FD include renal failure, cardiomyopathy, and early cerebrovascular events. However, ocular lesions are often an early sign of the disease and can be detected in eye examinations [4]. One recent study showed that the most common ocular finding in FD is cornea verticillata, which is reported in up to $95 \%$ of male patients and $88 \%$ of female carriers [5]. Cataract was found in approximately $70 \%$ of male and $35 \%$ of females;

Received: March 14, 2018, Revised: April 5, 2018

Accepted: April 10, 2018

Corresponding Author: Yu Cheol Kim, Department of Ophthalmology, Keimyung University School of Medicine,

56, Dalseong-ro, Jung-gu, Daegu 41931, Korea

Tel: +82-53-250-8026, Fax: +82-53-250-7705

E-mail: eyedr@dsmc.or.kr tortuosity of conjunctival and retinal vessels was present in up to $77 \%$ of males and in approximately $20 \%$ of female patients. Extra-corneal lesions are almost always accompanied by cornea verticillata [4]. In Korea, there was a case of nine FD patients in one family presented with cornea verticillata. However there was no case report about the vessel abnormalities using various kinds of imaging modalities in de novo FD patient [6. Here, the authors report a case of confirmed FD without a positive family history (de novo mutation), who underwent ophthalmologic examination and showed cornea verticillata and conjunctival vessel abnormalities.

\section{CASE}

A 41-year-old Asian male diagnosed with FD visited the clinic for ocular evaluation. The patient had been healthy until the age of 28 years, except for mild hypohidrosis since childhood. At the age of 28 years, the proteinuria was detected in regular medical checkup. He underwent renal biopsy and diagnosed with FD. There was no apparent family history of FD. Therefore, genetic analysis was performed to family members of the patient (mother and older brother) and

Copyright (C) 2018 Yeungnam University College of Medicine

This is an Open Access article distributed under the terms of the Creative Commons Attribution Non-Commercial License (http://creativecommons.org/licenses/by-nc/4.0/) which permits unrestricted non-commercial use, distribution, and reproduction in any medium, provided the original work is properly cited. 
none of them showed any alteration in the GLA gene, however, the patient had a mutation in hemizygosis at nucleotide c.293 C>T, suggesting a de novo mutation in patient (Fig.

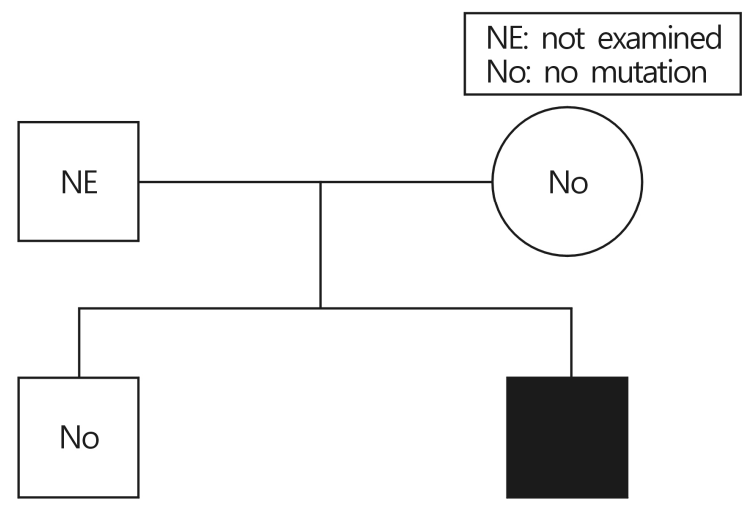

Heterozygote with c.293 C>T

(S297F) mutation

Fig. 1. The family tree of de novo Fabry disease. The patient had a mutation in hemizygosis at nucleotide c.293 C>T, this mutation was not detected from his mother and older brother.
1). He also had left ventricular hypertrophy and undergoing hemodialysis for chronic kidney disease. There was no history of systemic drug therapy that could develop vortex keratopathy. Slit lamp examination, fundus photography, fluorescein angiography, indocyanine green angiography (HRA-2; Heidelberg Engineering, Dossenheim, Germany), and optical coherence tomography (DRI OCT-1; Topcon, Tokyo, Japan) were performed. On examination, both eyes showed 20/20 best-corrected visual acuity and $14 \mathrm{mmHg}$ intraocular pressure. The slit lamp examination revealed a bilateral, paracentral, whorl-like pattern of white-to-brown corneal deposits (Figs. 2A, 2B). Tortuosity of episcleral vessels was noted in the right eye (Fig. 2C). Both eyes showed tortuosity and aneurysmal dilatation of the inferior bulbar conjunctival vessels (Figs. 2D, 2E). Cataract and retinal vessel tortuosity were not observed, and other imaging modalities demonstrated unremarkable findings (Figs. 2F, 3). As we considered the corneal and conjunctival abnormalites are the clinical signs of Fabry disease and the patient had been treated with enzyme
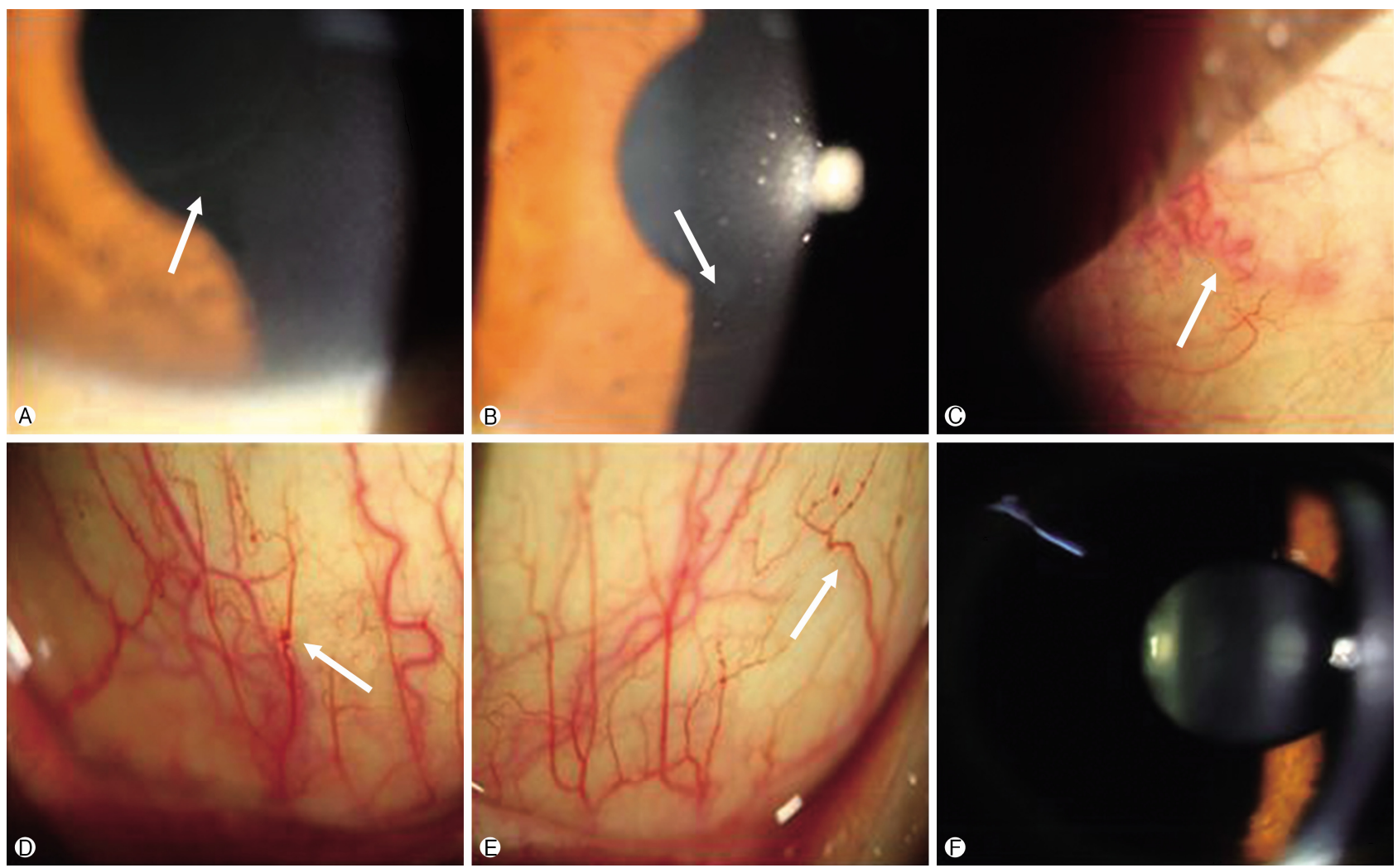

Fig. 2. Photographs of a patient with de novo Fabry disease. Cornea verticillata (arrow) show whorl-like pattern of white, brown corneal opacity in the right eye (A) and left eye (B). (C) Tortuosity and dilatation of episcleral vessel (arrow) of the right eye. Tortuosity and aneurysmal dilatation of the inferior bulbar conjunctiva vessels (arrow) in the right eye (D) and left eye (E). (F) Lens evalua- tion show no signs of Fabry cataract. 

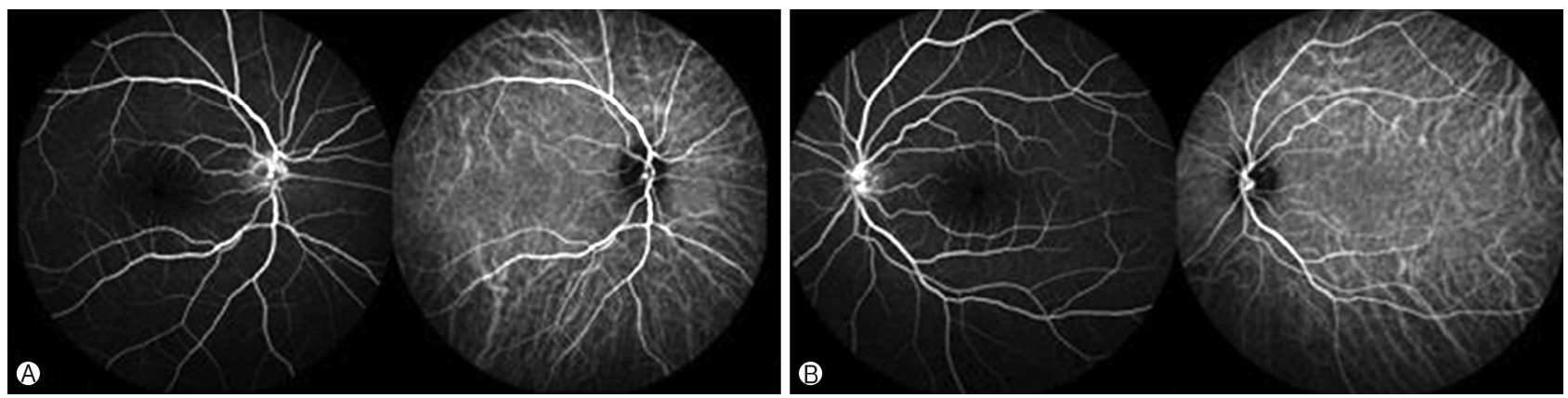

Fig. 3. Photographs of fluorescein angiography and indocyanine green angiography (A, right eye; B, left eye) taken about 2 minutes after the injection show no vascular abnormality.

replacement therapy (ERT), we decided to observe the ophthalmic findings.

\section{DISCUSSION}

FD is a rare X-linked hereditary disease. Due to its rarity, FD diagnosis remains a challenge. Furthermore, the diagnosis is even more difficult when family history is absent [7]. Delayed diagnosis of FD is still common; there is on average, a gap of a decade or more, between the onset of symptoms and diagnosis of FD. However, the ocular manifestations begin in childhood [4]. In one retrospective analysis from the United States; $26 \%$ of FD patients with no family history of the disease were diagnosed by an ophthalmologist, suggests that ophthalmic physicians can play an important role in diagnosis of FD [8].

In this case, a detailed ocular examination was performed to evaluate this patient with de novo FD. We identified cornea verticillata and tortuous, aneurysmal dilatation of the inferior bulbar conjunctival vessels on slit lamp examination. These findings support that the extra-corneal lesions are accompanied by cornea verticillata and the vascular abnormalities are most often seen on the inferior bulbar conjunctiva. However, other abnormalities such as Fabry cataract and retinal vessel disorders were not observed. There have been reports that corneal and conjunctival vascular abnormalities manifest at a younger age than lens or retinal disorders in FD [4]. The patient is on ERT and this may have slowed down disease progression.

This case highlights the fact that ophthalmologists should always be aware of the possibility of de novo FD in patients with corneal verticillata and the inferior bulbar conjunctival vessels abnormalities, even in the absence of a family history.

In conclusion, we report the case of de novo FD patient who underwent comprehensive ophthalmological evaluations using various kinds of imaging modalities and showed cornea verticillata and inferior conjunctival vessel abnormalities.

\section{CONFLICT OF INTEREST}

No potential conflict of interest relevant to this article was reported.

\section{ORCID}

You Hyun Lee, https://orcid.org/0000-0001-8116-7942

Yu Cheol Kim, https://orcid.org/0000-0003-1615-6651

\section{REFERENCES}

1. Terryn W, Cochat P, Froissart R, Ortiz A, Pirson Y, Poppe B, et al. Fabry nephropathy: indications for screening and guidance for diagnosis and treatment by the European Renal Best Practice. Nephrol Dial Transplant 2013;28:505-17.

2. Pisani A, Visciano B, Roux GD, Sabbatini M, Porto C, Parenti $\mathrm{G}$, et al. Enzyme replacement therapy in patients with Fabry disease: state of the art and review of the literature. Mol Genet Metab 2012;107:267-75.

3. Iemolo F, Pizzo F, Albeggiani G, Zizzo C, Colomba P, Scalia S, et al. De novo mutation in a male patient with Fabry disease: a case report. BMC Res Notes 2014;7:11.

4. Samiy N. Ocular features of Fabry disease: diagnosis of a treatable life-threatening disorder. Surv Ophthalmol 2008;53:41623.

5. Sher NA, Letson RD, Desnick RJ. The ocular manifestations in Fabry's disease. Arch Ophthalmol 1979;97:671-6.

6. Lee HG, Kim MJ, Choi CY, Tchah H. Fabry disease in a family: 
four patients and five carriers. J Korean Ophthalmol Soc 2006; 47:1496-501. Korean.

7. Pisani A, Daniele A, Di Domenico C, Nigro E, Salvatore F, Riccio E. Late diagnosis of Fabry disease caused by a de novo mutation in a patient with end stage renal disease. BMC Res
Notes 2015;8:711.

8. Branton MH, Schiffmann R, Sabnis SG, Murray GJ, Quirk JM, Altarescu G, et al. Natural history of Fabry renal disease: influence of alpha-galactosidase A activity and genetic mutations on clinical course. Medicine (Baltimore) 2002;81:122-38. 